\title{
Reflets
}

Revue d'intervention sociale et communautaire

\section{Que signifie être jeune adulte aujourd'hui? Les points de vue de jeunes adultes ayant reçu un diagnostic de maladie mentale}

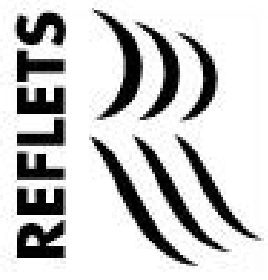

\section{Caroline Veilleux et Marc Molgat}

Volume 16, numéro 1, printemps 2010

Paternités méconnues (Deuxième partie)

URI : https://id.erudit.org/iderudit/044446ar

DOI : https://doi.org/10.7202/044446ar

Aller au sommaire du numéro

Éditeur(s)

Reflets : Revue d'intervention sociale et communautaire

ISSN

1203-4576 (imprimé)

1712-8498 (numérique)

Découvrir la revue

Citer cet article

Veilleux, C. \& Molgat, M. (2010). Que signifie être jeune adulte aujourd'hui? Les points de vue de jeunes adultes ayant reçu un diagnostic de maladie mentale. Reflets, 16(1), 152-179. https://doi.org/10.7202/044446ar

\section{Résumé de l'article}

Cet article est issu d'une étude qui s'inscrit dans une démarche d'exploration de la réalité plurielle actuelle des jeunes adultes ayant reçu un diagnostic de maladie mentale à travers la considération de leur discours. Il vise dans un premier temps à déterminer si ces jeunes adultes se perçoivent comme des adultes et à identifier les critères associés à cette perception d'être adulte. En second lieu, la recherche vise à circonscrire l'impact de la maladie mentale sur la perception de soi en tant que jeune adulte et à connaître la représentation et le positionnement de ces jeunes adultes par rapport aux services de santé mentale. Pour ce faire, des entrevues semi-dirigées ont été réalisées auprès de huit jeunes adultes âgés de 18 à 35 ans, qui ont reçu un diagnostic de maladie mentale. L'analyse approfondie du discours a permis de relever que ces jeunes adultes se réfèrent moins aux marqueurs de transition formels tels que l'entrée dans le marché du travail et la parentalité, pour expliquer leur parcours de vie et s'en remettent davantage à leurs expériences personnelles de vie. Ils se réfèrent à de nouveaux repères pour ainsi donner un sens à leur réalité, ce qui a comme conséquence de préserver en partie l'image de soi. Quelques pistes de réflexion pour l'intervention sont finalement proposées.

Tous droits réservés ( $)$ Reflets : Revue d'intervention sociale et communautaire, Ce document est protégé par la loi sur le droit d'auteur. L’utilisation des 2010 services d'Érudit (y compris la reproduction) est assujettie à sa politique d'utilisation que vous pouvez consulter en ligne.

https://apropos.erudit.org/fr/usagers/politique-dutilisation/ 


\title{
Que signifie être jeune adulte aujourd'hui? Les points de vue de jeunes adultes ayant reçu un diagnostic de maladie mentale
}

\author{
Caroline Veilleux \\ Travailleuse sociale
}

Marc Molgat

Professeur, École de service social, Université d'Ottawa

\section{Résumé}

Cet article est issu d'une étude qui s'inscrit dans une démarche d'exploration de la réalité plurielle actuelle des jeunes adultes ayant reçu un diagnostic de maladie mentale à travers la considération de leur discours. Il vise dans un premier temps à déterminer si ces jeunes adultes se perçoivent comme des adultes et à identifier les critères associés à cette perception d'être adulte. En second lieu, la recherche vise à circonscrire l'impact de la maladie mentale sur la perception de soi en tant que jeune adulte et à connaitre la représentation et le positionnement de ces jeunes adultes par rapport aux services de santé mentale. Pour ce faire, des entrevues semi-dirigées ont été réalisées auprès de huit jeunes adultes âgés de 18 à 35 ans, qui ont reçu un diagnostic de maladie mentale. L'analyse approfondie du discours a permis de relever que ces jeunes adultes se réferrent moins aux marqueurs de transition formels tels que l'entrée dans le marché du travail et la parentalité, pour expliquer leur parcours de vie et s'en remettent davantage à leurs expériences personnelles de vie. Ils se réferent à de nouveaux 
repères pour ainsi donner un sens à leur réalité, ce qui a comme conséquence de préserver en partie l'image de soi. Quelques pistes de réflexion pour l'intervention sont finalement proposées.

Mots clés : jeune adulte, maladie mentale, perception de soi, image de soi, réalité adulte, marqueur de transition

\section{Abstract}

Exploratory research reported in this article investigates the plural reality of today's young adult diagnosed with a mental illness. One of the purposes of this research is to verify if these young adults see themselves as adults, and identify which factors influences this perception. Another objective is to understand the impact of mental illness on young adults self perception as well as their representation and positioning toward mental illness services. Eight young adults diagnosed with a mental illness were interviewed for this research. The results of a thorough discourse analysis show that these young adult refer more to personal life experience than to formal transition marks in order to explain their life path. Possible venues for intervention are also discussed.

Keywords : Young adult, mental illness, self perception, being adult, transition mark

\section{Introduction}

Les personnes ayant franchi le cap de leurs vingt ans et ayant reçu un diagnostic de maladie mentale se considèrent-elles comme des adultes à part entière? Alors qu'aujourd'hui la psychiatrie oriente son intervention afin que les personnes atteintes de maladies mentales puissent vivre dans la communauté et non en institution, cela signifie-t-il pour autant que ces personnes ont l'impression de vivre de manière autonome et indépendante? Cette question se pose avec d'autant plus acuité pour les jeunes vivant avec des problèmes de santé mentale et qui ont l'âge de vivre les transitions 
qui mènent habituellement à la vie adulte. Les enjeux que pose la maladie mentale dans les trajectoires de vie de ces jeunes adultes apparaissent comme étant non négligeables, notamment en regard de la perception de soi comme un être autonome et indépendant, perception qui se construit non seulement sur les conditions de vie des personnes, mais aussi sur leur estime de soi, leurs capacités d'adaptation, le regard des autres et les discours sociaux (et médicaux) concernant l'intégration sociale et la maladie mentale.

Cet article tente de répondre à ce questionnement dans un contexte où, pour la plupart des jeunes, les événements qui marquent le passage à la vie adulte telles la décohabitation familiale, l'entrée dans le marché du travail, la formation d'un couple et la parentalité ne forment plus un parcours linéaire (RoulleauBerger, 1999; Gauthier, 2000) et sont reportés dans le temps. La singularité des trajectoires et la réversibilité de certains marqueurs de transition ainsi que leur désynchronisation (Galland, 2004), s'expliqueraient aujourd'hui par de multiples facteurs comme la précarité socioéconomique, les nouveaux rapports à la famille, les exigences du marché du travail ou encore, par l'état de santé physique ou mentale d'une personne. Ces parcours à la fois individualisés et pluriels des jeunes poussent à la réflexion et entraînent de nombreux questionnements au sujet de leur définition de soi. Comment arrivent-ils à se considérer comme des adultes dans un contexte de report des moments de transitions et de diversité et de singularité des parcours? Ont-ils l'impression de vivre une sorte d'entre-deux, pas encore tout à fait adulte, mais pas non plus encore adolescent? Et qu'en est-il alors des jeunes vivant avec un problème de santé mentale? Vivraient-ils de la même manière ces hésitations à se dire adulte?

Ces interrogations mettent en relief l'importance de mieux comprendre comment la santé mentale peut moduler ou teinter la définition de soi des jeunes adultes. Cette compréhension nous semble essentielle si l'on souhaite replacer les jeunes adultes utilisateurs des services de santé mentale au centre même des interventions et services (Rodriguez, Poirel et Corin, 2001) et leur donner un espace de parole pour exposer le plus fidèlement 
possible leurs points de vue, leurs valeurs, leurs défis et leurs préoccupations. La recherche sur laquelle se fonde cet article visait ainsi à explorer la réalité des jeunes adultes ayant reçu un diagnostic de maladie mentale, à partir de leur propre discours sur leurs perceptions d'être ou non des adultes et la place de la maladie mentale et des services dans ces représentations ${ }^{2}$.

L'article présentera d'abord les éléments d'une problématique sur la définition de la perception de soi comme adulte en lien avec la maladie mentale. Puis, nous exposons la méthodologie et l'échantillon de la recherche avant de présenter les résultats qui portent sur la perception d'être adulte des interviewés, les réalités auxquelles ils font face et leurs représentations de la maladie mentale et des services auxquels ils ont accès.

\section{Jeunesse et définition de soi comme adulte}

"Conséquence

du prolongement des études et des difficultés d'insertion professionnelle notamment, les jeunes dans plusieurs pays occidentaux... ont de plus en plus reporté ces transitions dans le temps, ce qui a incité bon nombre de chercheurs à décrive la jeunesse en termes d'allongement..."
Longtemps, l'âge adulte a été défini par le statut social et les rôles associés à cet âge, tels le travail et la famille (Plug, Zeijl et Du Bois-Reymond, 2003), alors que la jeunesse a été dans l'imaginaire collectif, décrite par un statut dit transitoire (De Singly, 2000). Les jeunes devaient s'approprier un modèle de parcours de vie, un statut adulte définitif et des rôles associés à l'âge adulte. Par contre, vers la fin du XX $X^{\mathrm{e}}$ siècle, ce modèle de l'identification s'est dissipé pour laisser la place à un modèle basé sur l'expérimentation (Galland, 1990). On remet dès lors en question les catégorisations généralement attribuées aux jeunes (Schehr, 2000). Dorénavant, il devient essentiel de s'attarder plus sérieusement à la pluralité des expériences des jeunes adultes tout en ne négligeant pas les événements de vie majeurs propres à l'entrée dans la vie adulte.

On reconnait les nouveaux rapports qu'entretiennent les jeunes adultes avec les marqueurs de transitions comme la fin des études, le départ du domicile familial, l'insertion dans le marché du travail et la formation d'un couple. Conséquence du prolongement des études et des difficultés d'insertion professionnelle notamment, les jeunes dans plusieurs pays occidentaux, dont le Canada (Beaujot 
"Les jeunes adultes d'aujourd' hui font face à des enjeux majeurs liés au contexte socioéconomique, tels que le chômage, l'intellectualisation du travail, les restructurations du marché du travail et la précarisation de l'emploi, les discours sociaux ainsi que les phénomènes de désynchronisation des transitions et de prolongement des périodes de la vie..." et Kerr, 2007), ont de plus en plus reporté ces transitions dans le temps, ce qui a incité bon nombre de chercheurs à décrire la jeunesse en termes d'allongement (Cavalli et Galland, 1993). En même temps, les parcours des jeunes sont aussi moins linéaires, traduisant un agencement plus complexe et une plus grande réversibilité des transitions. Biggart et Walther (2006) réferent ainsi aux transitions yo-yo qui favorisent l'émergence de situations où les jeunes vivent du même coup comme des adolescents et comme des adultes, tantôt réticents face à l'engagement, tantôt assumant plusieurs responsabilités à la fois.

Cette situation crée des processus de socialisation des jeunes qui sont différents de ceux qui existaient il y a quelques décennies. Les jeunes adultes d'aujourd'hui font face à des enjeux majeurs liés au contexte socio-économique, tels que le chômage, l'intellectualisation du travail (Roulleau-Berger, 1999), les restructurations du marché du travail et la précarisation de l'emploi (Gauthier, 1994; Molgat, 1999), les discours sociaux (Angenot, 1979; 1984) ainsi que les phénomènes de désynchronisation des transitions et de prolongement des périodes de la vie (Galland, 2004). Tous ces défis peuvent fragiliser la construction identitaire des jeunes (Roulleau-Berger, 1999), intervenir dans leurs démarches d'insertion (Molgat, 1999; Coleman et Husén, 1985) et précipiter l'apparition de risques potentiels de marginalisation et d'exclusion sociale (Schehr, 2000; Molgat, 2000). En ce sens, la réalité des jeunes adultes ne peut échapper à l'incertitude, ce qui a comme principale conséquence de présentifier leur existence et par le fait même, d'occasionner des difficultés à se projeter dans le futur (Leccardi, 2006). De ce point de vue, les jeunes adultes tenteraient de se construire une identité à partir de repères parfois vaporeux et de tracer leur propre chemin vers l'avenir. Une sorte de questionnement existentiel dépasserait ainsi l'adolescence pour s'étendre chez les jeunes adultes (Nakkula et Toshalis, 2006), et renverrait à une forte tendance à expérimenter de multiples choix et possibilités et au désir de se sentir "soi-même " (Anatrella, 1999).

Comment alors se définir comme adulte dans une société où les points de repère qui caractérisent ce statut sont devenus 
"Ce n'est que dans les études où l'on étudie les individus âgés vers la fin de la vingtaine qu'on voit s'accroître le nombre de ceux et celles qui se disent adultes à part entière..." flous? Cette question est importante dans la mesure où le fait de se considérer comme un adulte ou non aurait des répercussions majeures sur la réalité quotidienne, sur la participation active aux rôles sociaux et sur le développement futur des jeunes adultes (Reitzle, 2006). En tenant compte des changements que nous venons d'évoquer dans les pages précédentes, il est peu surprenant d'observer que la majorité des jeunes adultes âgés de 18 à 25 ans - et notamment ceux de moins de 20 ans - ne se considèrent pas comme des adultes à part entière (Nelson et McNamara Barry, 2005). En d'autres mots, bon nombre de jeunes adultes interviewés dans le cadre d'études récentes se considèrent adultes en partie seulement (Gaudet, 2005; Plug, Zeijl et Du Bois-Reymond, 2003). Ce n'est que dans les études où l'on étudie les individus âgés vers la fin de la vingtaine qu'on voit s'accroître le nombre de ceux et celles qui se disent adultes à part entière (Molgat, 2007).

Plusieurs auteurs ont tenté, en appliquant différentes méthodologies, de mettre en relief les critères associés à l'impression d'être adulte auprès de groupes de jeunes adultes. L'âge adulte a été défini en fonction de la pluralité des engagements dans diverses sphères de leur vie et en fonction du fait d'assumer de plus en plus de responsabilités jugées majeures aux yeux des jeunes adultes (Gaudet, 2005). L'impression d'être un adulte, totalement ou en partie, renvoie également à des dimensions de l'identité adulte liées aux notions de responsabilité, d'autonomie et d'indépendance financière (Arnett, 1997; 1998; Molgat, 2007). Chez les individus âgés de 18 à 25 ans, et surtout chez les plus jeunes dans cette catégorie d'âge, l'âge adulte se révèle davantage associé à des dimensions individuelles telles que la maturité et l'autonomie qu'à des marqueurs de transition comme la fin des études et l'entrée dans le marché du travail (Arnett, 1997; 1998; Plug, Zeijl et Du Bois-Reymond, 2003; Westberg, 2004).

Or, lorsque les recherches portent sur des personnes de plus de 25 ans, les marqueurs de transition apparaissent comme des moments significatifs de la définition de soi comme adulte (Molgat, 2007). Ces différences de perception reflètent le fait d'avoir vécu des transitions. Chez les 18 à 20 ans, plusieurs n'ont fait aucune transition alors qu'aux yeux des 25-29 ans qui les 
ont vécues, celles-ci apparaissent comme des moments qui ont favorisé l'émergence et concrétisé des sentiments d'autonomie et d'indépendance. En ce sens, les recherches indiquent que les transitions en lien avec la sphère familiale, notamment la décohabitation parentale, la mise en couple et surtout la parentalité, demeurent fortement associées à l'impression d'être un adulte, particulièrement lorsqu'elles ont été expérimentées (Gaudet, 2005; Molgat, 2007; Shanahan et collab., 2005; Westberg, 2004). Enfin, il faut souligner que l'appartenance à certaines catégories sociodémographiques, telles que la classe sociale et le genre, influencerait, bien que modérément, la conception de l'âge adulte de certains jeunes adultes (Bidart, 2005; Plug, Zeijl et Du BoisReymond, 2003).

\section{Se dire adulte en situation de maladie mentale}

L'ensemble de ces constats au sujet des dimensions ayant une incidence sur la perception de soi comme adulte, invite à s'interroger sur cette perception chez celles et ceux qui sont aux prises avec une situation de maladie mentale. La maladie mentale, telle que conçue dans la présente problématique, renvoie à une altération de la pensée, de l'humeur et/ou du comportement qui entraîne une importante détresse psychologique et un dysfonctionnement observé dans une ou plusieurs sphères de la vie de la personne (Santé Canada, 2002). La perception de soi d'un jeune adulte vivant avec une maladie mentale comprendrait des repères développementaux et sociologiques en lien avec le contexte social dans lequel il se retrouve actuellement. Les enjeux que pose la maladie mentale dans sa trajectoire de vie apparaissent comme étant non négligeables, et ce, particulièrement au niveau de l'estime de soi, de la capacité d'adaptation, du regard des autres et de l'influence des discours sociaux et médicaux dans la trajectoire personnelle. Ces enjeux pourraient en fait nuire à la capacité de ces jeunes à se concevoir comme adultes à part entière. 
"Cette image de soi plutôt négative chez les jeunes adultes ayant reçu un diagnostic de maladie mentale entraîne des répercussions au niveau des relations interpersonnelles et des habiletés sociales."
Les recherches montrent en effet que la présence d'une maladie mentale est fréquemment associée à une diminution généralisée de l'estime de soi (Alie, Boisvert et Brodeur, 2005; Gralinski-Bakker et collab., 2005) et porte souvent atteinte à la construction d'une identité positive (Desmarais et collab., 2000); elle mène à des difficultés persistantes à maintenir une image de soi et une identité autre que celle reliée au statut de patient psychiatrique (Poirier et Gagné, 1988). Cette image de soi plutôt négative chez les jeunes adultes ayant reçu un diagnostic de maladie mentale entraîne des répercussions au niveau des relations interpersonnelles et des habiletés sociales. En effet, cette tendance à l'autodévalorisation porte ces jeunes à se replier, à se recentrer sur eux-mêmes, sur leurs difficultés et leur maladie mentale, cette dernière devenant bien souvent une des seules qualifications qu'on leur reconnait (Alie, Boisvert et Brodeur, 2005).

Comme la maladie mentale prend de plus en plus de place dans la définition de soi, elle compromet aussi les mécanismes d'adaptation des jeunes. Une étude relève ainsi que les jeunes adultes vivant avec d'importantes difficultés psychologiques utilisent des stratégies d'autorégulation moins adaptées et moins efficaces que celles rencontrées chez les jeunes adultes sans difficulté de cet ordre (Shulman et collab., 2009). De plus, leur état de santé mentale peut modifier la façon dont ils se projettent dans le futur et la façon dont ils entrevoient leur avenir. Plus spécifiquement, Nurmi (1999) a identifié qu'un état dépressif chez les jeunes peut altérer leur perception d'eux-mêmes et la perception qu'ils ont de leur propre développement ainsi que la définition des buts personnels à long terme. Les distorsions cognitives inhérentes à un état dépressif et entre autres liées à la dépréciation, la dichotomie et le fatalisme pourraient expliquer l'altération des perceptions de ces jeunes.

Le regard extérieur porté sur la maladie mentale et sur la personne qui en est atteinte constitue un autre facteur déterminant dans la manière dont se perçoit le jeune adulte. La maladie mentale provoque beaucoup de réactions, tant dans l'entourage immédiat de la personne que dans la sphère publique, comme dans les médias. Les réactions de peur, les manifestations de rejet, les attitudes de discrimination, les préjugés, les mécanismes de 
"...le jeune adulte qui a reçu un diagnostic de maladie mentale fait face à la fois aux discours sociaux dominants préconisant l'activité, l'efficacité et l'autonomie, et au discours médical dominant, qui offre un cadre sécurisant de maîtrise de la souffrance. Un des plus grands enjeux auquel se heurtent ces jeunes adultes concerne le décalage marqué entre les exigences du marché du travail et leurs conditions personnelles de vie. marginalisation et d'exclusion sociale (Poulin et collab., 1994) traduisent généralement une incompréhension, une ignorance. Les jeunes adultes transigent régulièrement dans leurs rapports aux autres, avec ces représentations de la maladie mentale, qui occupent beaucoup de place dans leur quotidien, tel que l'illustre l'étude de Danielle Desmarais et ses collaborateurs (2000) :

À leur avis [c'est-à-dire de l'avis des jeunes], les
préjugés tendent à s'accentuer quand il s'agit d'une
population de jeunes adultes psychiatrisés puisqu'on
associe généralement la jeunesse au fait d'avoir son
plein potentiel, d'être performant, d'avoir tout l'avenir
devant soi. [...] Bref, ces jeunes sont confrontés aux
mêmes paramètres d'entrée dans la vie adulte que tous
les autres jeunes, mais ils doivent en plus mener un
combat quotidien contre le trouble mental. (p.69)

Sur un registre plus large encore, le jeune adulte qui a reçu un diagnostic de maladie mentale fait face à la fois aux discours sociaux dominants préconisant l'activité, l'efficacité et l'autonomie, et au discours médical dominant, qui offre un cadre sécurisant de maitrise de la souffrance (Desmarais et collab., 2000). Un des plus grands enjeux auquel se heurtent ces jeunes adultes concerne le décalage marqué entre les exigences du marché du travail et leurs conditions personnelles de vie. Ce que le marché du travail demande en matière de performance, de productivité, d'efficacité et d'initiative s'oppose aux capacités, désormais limitées, de ces jeunes à jouer différents rôles sociaux (Desmarais et collab., 2000), dont celui de travailleur ou d'employé. Le contexte actuel du marché du travail permet difficilement la modification des temps de travail individuel et se montre peu avenant en ce qui concerne l'adaptabilité des tâches aux conditions particulières de santé mentale des employés ou dans la prestation d'assurances en cas de maladie ou d'invalidité. La possibilité de se réaliser à travers l'emploi apparait ainsi compromise temporairement ou carrément remise en question, ce qui laisse présager un effet plus négatif sur l'image de soi du jeune adulte. 
Outre le marché du travail, tout l'engrenage institutionnel, particulièrement sur le plan de l'organisation des services dans le champ de la santé mentale, contribue à sa façon à déformer l'image de soi du jeune adulte. Le champ de la santé mentale a une capacité de déculpabiliser l'individu de toute charge ou faute personnelle dans l'explication de l'apparition de la maladie, mais en revanche, cette déresponsabilisation se confronte au processus identitaire du jeune adulte. Poirier et Gagné (1988) résument bien l'impact de l'univers psychiatrique sur l'existence du jeune adulte : "Cette tendance à l'infantilisation et à la déresponsabilisation des patients est en contradiction fondamentale avec les acquis que doit faire le jeune adulte psychiatrisé pour pouvoir s'insérer dans la société.» (p.139). En le prémunissant ou plutôt, en le dépossédant de toute responsabilité, on lui indique qu'il n'est pas en mesure de prendre soin de lui-même, de pouvoir agir et penser librement et de pouvoir bénéficier d'une autonomie et d'une indépendance alors que les jeunes adultes, dans leurs démarches de connaissance

"Le discours médical ébranle en quelque sorte la perception de soi dans la manière fataliste d'aborder les limitations d'ordre affectif, psychologique et social. " et d'affirmation de soi s'orientent dans la direction opposée. Le discours médical ébranle en quelque sorte la perception de soi dans la manière fataliste d'aborder les limitations d'ordre affectif, psychologique et social. Estroff (1989) rattache même l'expérience de la maladie mentale à une perte du soi, d'une identité positive et de rôles sociaux, perte qui aggrave davantage l'état de la personne lorsque la maladie se chronicise. La construction identitaire du jeune adulte rivalise en ce sens avec l'approche déshumanisante de la psychiatrie qui déstructure ou fragmente le soi, et ce, malgré les efforts acharnés des professionnels de mettre au centre de leur pratique le processus global de la personne. Ce constat pourrait être en lien avec le phénomène répandu de non-consultation psychiatrique observé chez les jeunes adultes (Biddle et collab., 2007).

En considérant la manière dont ces enjeux peuvent intervenir dans la perception d'être ou non un adulte chez les jeunes qui ont un problème de santé mentale diagnostiqué, il nous parait important de rendre compte de la perspective qu'en ont les premiers intéressés. À cette fin, les discours des jeunes adultes ayant reçu un diagnostic de maladie mentale que nous avons 
rencontrés en cours de recherche présentent la possibilité de comparer les perceptions de ces personnes avec les résultats des recherches portant sur les jeunes adultes en général, que nous avons présentés dans les pages précédentes. L'analyse de ces discours se révèle d'autant plus intéressante que les personnes que nous avons rencontrées sont âgées de plus de 25 ans, c'est-à-dire qu'elles sont à un âge où le sentiment d'être adulte s'est généralement concrétisé pour une grande partie de la population.

\section{Méthodologie et échantillon}

Une méthodologie qualitative a été utilisée dans ce projet de recherche exploratoire visant à décrire les perceptions des jeunes adultes ayant un diagnostic de maladie mentale et le sens qu'ils accordent à leurs expériences (Veilleux, 2009). Des entretiens semi-structurés ont été réalisés, retranscrits textuellement et analysés à l'aide d'une grille d'analyse individuelle (L'Écuyer, 1987). Le guide d'entrevue semi-structuré, tout comme le questionnaire portant sur les caractéristiques sociodémographiques des participants, a permis d'aborder de nombreux thèmes avec les participants, dont leur perception de ce que signifie généralement être un jeune adulte, ainsi que leur impression d'être ou non un adulte, tant du point de vue personnel, relationnel et professionnel que socioéconomique et politique. Le guide d'entretien permettait également aux répondants de se prononcer sur leurs défis, les obstacles qu'ils rencontrent, leurs plans futurs et la manière dont les services de santé mentale tiennent compte (ou non) de la réalité des jeunes adultes. De surcroît, un questionnaire, administré oralement, portant sur les caractéristiques sociodémographiques des participants, a permis de dresser un profil général des interviewés ${ }^{3}$.

Le processus d'analyse des données qui s'inspire de l'approche thématique de type mixte (Miles et Huberman, 2003) comprend une codification à double entrée, c'est-à-dire une première codification à partir des propos des participants et une seconde, 
à partir d'un repérage de thèmes issus de la recension des écrits. L'analyse des données s'appuie sur la théorisation ancrée (grounded theory) (Glaser et Strauss, 1967), en raison de la nature exploratoire de la recherche et de la nécessité d'avoir un cadre d'analyse flexible. Le fait d'alterner entre la collecte et l'analyse des données permet d'établir des repères théoriques qui reflètent plus fidèlement le discours des participants. L'analyse a donc été complétée en appliquant un mode itératif qui a nécessité plusieurs lectures des transcriptions d'entrevues (Contandriopoulos et collab., 1990).

L'échantillon est composé de huit participants, dont cinq femmes et trois hommes âgés de 26 à 35 ans. La majorité d'entre eux sont âgés de 26 à 30 ans $(5 / 8)$ alors que les autres sont âgés de 32 ans $(1 / 8)$ et de $35(2 / 8)$ ans. La quasi-totalité des participants $(7 / 8)$ sont célibataires au sens légal du terme, bien que la moitié d'entre eux aient un partenaire amoureux; aucun n'a d'enfant. Trois répondants habitent en appartement (seul ou en colocation) et trois autres dans un foyer d'accueil; les deux autres répondants occupent leur logement en tant que locataires d'un appartement subventionné ou comme propriétaire. En ce qui a trait au niveau de scolarité, trois détiennent un diplôme d'études secondaires, deux ont complété un programme d'études collégiales et trois autres ont un diplôme d'études universitaires (de premier ou deuxième cycle). Pour ce qui est de l'emploi du temps, trois de ces jeunes adultes suivent actuellement un programme d'insertion socioprofessionnelle, deux travaillent à temps plein ou à temps partiel, deux sont aux études et un entreprend un retour aux études. Reflétant cette diversité d'occupations, les sources de revenus sont multiples : prestations de la sécurité du revenu (4/8), prestations rattachées aux mesures d'insertion socioprofessionnelle $(3 / 8)$, salaire $(2 / 8)$, prêts et bourses étudiants $(2 / 8)$ et prestations d'assurance $(1 / 8)$.

En ce qui concerne la santé mentale, trois différents diagnostics de maladie mentale ont été identifiés parmi les participants, soit la schizophrénie (4/8), le trouble dépressif majeur (3/8) et le trouble d'anxiété généralisé (1/8). L'échantillon se divise en deux grands spectres diagnostiques soit le spectre psychotique regroupant les quatre personnes dont le diagnostic est la schizophrénie et le 
spectre dépressif (la personne ayant un trouble d'anxiété généralisé a un passé de dépression majeure). Par ailleurs, quatre d'entre eux bénéficient de l'aide d'un psychiatre alors que les quatre autres reçoivent le support d'un médecin omnipraticien. En plus de cette aide médicale, certains ont recours aux services d'un psychologue en pratique privée (4/8) tandis que d'autres ont entrepris un suivi individuel auprès d'un intervenant dit communautaire (4/8).

L'analyse des entretiens nous amène à présenter les résultats qui suivent. En lien avec les questions posées, les résultats se regroupent sous trois grands volets, soit la perception d'être adulte, le contexte personnel de vie ainsi que la perception de soi comme adulte et la maladie mentale.

\section{Résultats}

\section{La perception d'être adulte}

La majorité des jeunes adultes que nous avons rencontrés se perçoivent seulement en partie comme des adultes. Ce résultat reflète sensiblement la réalité d'un bon nombre de jeunes adultes sans problème de santé mentale âgés de 18 à 25 ans (Nelson et McNamara Barry, 2005), mais ne concorde pas avec les recherches effectuées auprès d'individus plus âgés. Dans l'optique de bien distinguer les critères associés au fait de se percevoir comme un adulte de ceux associés au fait de ne pas se considérer comme un adulte, ceux-ci seront abordés séparément.

Lorsque les participants ont évoqué les critères associés à leur perception d'être adulte, les caractéristiques individuelles telles que la responsabilisation, l'autonomie, la maturité et la liberté prédominaient dans leurs discours, alors que les marqueurs de transition liés à l'entrée dans la vie adulte étaient peu souvent mentionnés. Or, cette perspective changeait lorsque nous leur demandions d'indiquer à quel moment dans leur parcours de vie respectif ils avaient eu l'impression de devenir adultes. En 
effet, l'émergence de la perception d'être adulte coïncide avec des moments clés dans leur vie. Ainsi, la moitié des participants ont relié la perception d'être adulte à un marqueur de transition, soit le départ du nid familial, la mise en couple ou l'entrée dans le marché du travail. Pour l'autre moitié de l'échantillon, la perception d'être adulte coïncide avec le début d'une période de stabilité dans l'expérience de la maladie mentale. En ce sens, contrairement aux résultats obtenus en lien avec la perception d'être adulte, la question du moment se démarque des tendances générales propres aux jeunes adultes sans problème de santé mentale et montre que l'expérience de la maladie mentale (selon les difficultés personnelles et les limitations fonctionnelles associées) peut modifier les repères sociaux et culturels marquant le passage de l'adolescence à l'âge adulte.

Les critères associés au fait de ne pas se percevoir comme un adulte sont, pour la majorité des participants, liés à l'absence de certains marqueurs de transitions comme le fait de ne pas avoir d'enfant ou le fait de ne pas avoir une propriété résidentielle. Le discours d'une participante illustre clairement cette perception :

Des fois, je me sens comme une adolescente, parce que je regarde les gens de mon âge qui sont déjà casés, qui ont un métier, une maison, des enfants. Pis moi, je suis là, je suis encore dans mes projets aussi flous, des relations amoureuses aussi floues. Tout ça fait que je dois encore être adolescente, je me sens pas adulte dans ce temps-là. (P4)

"Le fait de ne pas avoir expérimenté certains marqueurs de transition représente, pour plusieurs d'entre eux, un manque ou un frein dans l'atteinte d'un statut d'adulte. »
Le fait de ne pas avoir expérimenté certains marqueurs de transition représente, pour plusieurs d'entre eux, un manque ou un frein dans l'atteinte d'un statut d'adulte. À leurs yeux, ils ne peuvent se considérer pleinement comme des adultes en raison de l'absence de marqueurs de transition, alors qu'ils se montrent beaucoup plus nuancés lorsqu'il est question de leur définition générale de l'âge adulte. De façon évidente, les jeunes adultes interviewés conçoivent l'âge adulte sous un angle beaucoup plus large, plus holistique lorsque la question ne les touche pas directement. 


\section{Le contexte personnel de vie}

Afin de mieux comprendre le contexte dans lequel se forgent ces perceptions d'être ou non adulte, nous avons cherché dans l'entretien à élargir la réflexion à différentes dimensions de leur vie, à savoir leur travail ou occupation professionnelle, leur réseau social et leurs défis actuels et futurs.

\section{Le travail}

Sur le plan professionnel, les jeunes adultes que nous avons rencontrés se démarquent de leurs pairs dans la manière de concevoir le travail. Le rapport qu'entretiennent les jeunes adultes en général avec le travail et l'occupation a aujourd'hui une dimension plus utilitariste. La définition de soi par le travail et la valeur donnée à la sécurité d'emploi perdrait du terrain chez les jeunes (Hamel, 2000), laissant ainsi une plus grande place à la valorisation des relations interpersonnelles, des loisirs et des projets personnels. De ce point de vue, le travail apparait moins comme une occasion de s'accomplir, de développer une identité professionnelle ou d'éprouver un sentiment de réussite, mais davantage comme une source de revenus qui garantit une certaine autonomie et une indépendance financière permettant de se réaliser à l'extérieur du travail (Gendron et Hamel, 2004). Or, nous ne retrouvons pas ce rapport utilitariste au travail chez les jeunes adultes interviewés. En effet, ces derniers se concentrent davantage sur le plaisir et les sentiments d'accomplissement et

«De toutes les questions discutées au moment de l'entretien, celle du travail est centrale, non pas au sens de la performance et de l'ambition, mais plutôt au sens de l'accomplissement et de la réalisation de soi.» d'utilité que leur procure la réalisation de tâches et d'activités liées au travail. Cette différence peut s'expliquer par la situation psychologique des participants, qui ne leur permet pas de toujours répondre adéquatement aux demandes et exigences du marché du travail. De ce fait, les jeunes adultes interviewés redirigent leurs efforts vers des champs d'intérêt plus personnalisés. Ils redéfinissent par le fait même leurs propres idéaux professionnels et occupationnels à partir de ces champs d'intérêt, et non selon des attentes et des normes sociales. De toutes les questions discutées au moment de l'entretien, celle du travail est centrale, non pas au sens de la performance et de l'ambition, mais plutôt au sens de 
l'accomplissement et de la réalisation de soi. Ainsi, le travail est prioritaire pour ces jeunes adultes (au même titre que peuvent l'être la famille et le cercle d'amis) et revêt une valeur beaucoup plus expressive qu'instrumentale.

\section{Le réseau de soutien}

Nous avons aussi interrogé les participants sur la constitution et la qualité de leur réseau de soutien. Pour la majorité des jeunes adultes, le cercle d'amis forme la principale source de soutien et se caractérise par sa stabilité et la réciprocité des relations entre ses membres. Le fait que ces relations amicales aient autant d'importance dans le réseau social semble relié à l'absence de certains marqueurs de transition comme la parentalité ou la relation conjugale (Charbonneau, 2004). En effet, comme les jeunes adultes interviewés n'ont pas d'enfants à charge, donc aucune responsabilité parentale, ces derniers peuvent s'investir davantage dans leurs relations de nature amicale et sociale, notamment en leur consacrant plus de temps. Au sujet des relations familiales, les jeunes adultes qui disent entretenir des rapports sains et égalitaires avec leurs parents sont perçus davantage comme des adultes par ces derniers. Nous ne pouvons clairement déterminer à partir des entretiens les raisons pour lesquelles ces parents ont un rapport "d'adulte à adulte » avec leurs enfants, mais il est clair que ces répondants entretiennent une image plus positive d'eux-

"...les jeunes adultes qui disent entretenir des rapports sains et égalitaires avec leurs parents sont perçus davantage comme des adultes par ces derniers. » mêmes que les jeunes adultes dont les parents les considèrent essentiellement comme des enfants. Enfin, soulignons que même si les relations avec les parents s'avèrent plus ou moins satisfaisantes, les relations avec la fratrie tendent à s'améliorer avec le temps.

\section{Les défis actuels et futurs}

Les personnes que nous avons rencontrées ont évoqué les défis reliés à leur parcours académique et à l'insertion professionnelle, à leurs relations interpersonnelles, plus spécifiquement amoureuses et familiales, ainsi qu'à leur situation financière. De leur point de vue, ces défis sont liés aux conséquences de leur maladie mentale, parfois décrites comme envahissantes, telles que les problèmes de concentration et d'attention liés à l'usage de la médication, les 
"...tous les

participants ont $d \hat{u}$ remettre en question leurs choix de vie, abandonner certains itinéraires ou encore, adapter leurs choix en fonction de leurs conditions de vie associées à la maladie mentale.» difficultés à respecter une routine quotidienne et à s'engager dans des projets à long terme, l'instabilité émotionnelle ou la grande vulnérabilité au stress. Ces défis influencent la manière dont ces jeunes adultes se projettent dans l'avenir même si leurs projets se rapprochent grandement de ceux de jeunes adultes n'ayant pas de maladie mentale.

Ainsi, dans l'ensemble, les jeunes adultes interviewés veulent avoir des enfants et veulent concilier le travail, les loisirs, le couple et la famille. Ils connaissent les grandes lignes de leur projet de vie, mais ignorent encore le chemin par lequel ils vont s'y rendre, ce qui rejoint la tendance à la "présentification " de l'existence discutée précédemment. Cependant, l'expérience de la maladie mentale chez ces jeunes adultes a un impact sur la définition prospective de leur vie. À un certain moment de leur existence, tous les participants ont dû remettre en question leurs choix de vie, abandonner certains itinéraires ou encore, adapter leurs choix en fonction de leurs conditions de vie associées à la maladie mentale. Les propos d'un participant pour qui le parcours personnel et professionnel a complètement bifurqué reflètent ce constat lié à la conception renouvelée de l'avenir : «C'est sûr que l'avenir me parait beaucoup plus lourd. Et je m'efforce de le voir aussi joyeux. [...] C'est pas parce que tu n'as pas le même plan de maison qu'au début que ta maison ne tiendra pas debout. " (P2). À l'instar de ce participant, les jeunes adultes interviewés persistent à voir en l'avenir une lueur d'espoir, et ce, malgré leurs limites personnelles.

\section{La perception de soi comme adulte faisant face à un diagnostic de maladie mentale}

Nous abordons maintenant le troisième thème, soit la perception de soi comme adulte à travers l'expérience de la maladie mentale. L'analyse de ce thème permet en fait de présenter la manière dont les jeunes adultes prennent distance de la maladie mentale, à la fois dans leur définition de soi et dans leurs perspectives sur les services qui leur sont offerts. Ainsi, bien que la maladie mentale paraisse globalement freiner le processus de la définition de soi comme adulte, elle ne subsume pas complètement l'image de soi des jeunes 
que nous avons rencontrés. Cette dynamique apparaît clairement à l'analyse de sous-thèmes, soit la conception de la maladie mentale, le dévoilement du diagnostic (et le rapport aux autres) ainsi que la représentation des services de santé mentale. Les deux premiers sous-thèmes permettent de connaitre le positionnement des jeunes adultes par rapport à la maladie mentale et l'impact de la maladie sur leur image de soi, tandis que le dernier permet de mettre en évidence leurs points de vue au sujet des services de santé mentale et leurs propositions en vue de les modifier.

\section{La conception de la maladie mentale}

La conception que les jeunes adultes interviewés ont de la maladie émerge d'un contexte de vie spécifique où chacun y accorde sa propre signification, en fonction de ses expériences, de ses priorités personnelles ou encore de sa capacité d'introspection. Par exemple, pour un participant, la maladie symbolise l'échec, la faiblesse, et renvoie à une dynamique d'infantilisation. Bien qu'il se soit perçu comme un adulte actif et autonome dès l'âge de dix-sept ans, l'apparition soudaine de sa maladie lui est apparue comme une régression sur le plan développemental. Pour un autre participant, dont la définition idéale de la vie se résume au travail, la maladie signifie l'anormalité et le handicap puisqu'elle ne lui permet pas de répondre aux exigences du marché de l'emploi. Enfin, un autre participant met à distance la conception médicale de la maladie mentale en remettant en question la validité du diagnostic qu'il a reçu, en considérant que ce diagnostic correspond plus ou moins à l'ensemble de son vécu. Pour ce jeune adulte, sa maladie mentale

"Ces conceptions de la maladie montrent bien qu'elles sont intégrées de diverses manières à l'image que se font les répondants d'euxmêmes et qu'en fait, elles n'occupent pas toute la place dans la définition de soi..." dépasse un cadre d'explication pathologique et réfere plutôt à une série de difficultés personnelles et d'expériences familiales pénibles, voire traumatisantes.

Ces conceptions de la maladie montrent bien qu'elles sont intégrées de diverses manières à l'image que se font les répondants d'eux-mêmes et qu'en fait, elles n'occupent pas toute la place dans la définition de soi; au contraire, elles se superposent à d'autres dimensions de la perception de soi en tant qu'adulte ou non, en tant que travailleur ou en tant que personne lésée par des expériences familiales difficiles. 


\section{Le rapport aux autres}

Le sous-thème du rapport aux autres permet aussi de reconsidérer comment la maladie mentale des jeunes adultes interviewés a un effet sur leur perception d'eux-mêmes. Le rapport à l'autre se manifeste notamment dans la décision, cruciale pour plusieurs répondants, de dévoiler le diagnostic à d'autres. Cela permet d'informer sur le processus d'acceptation de la personne et sur la qualité et l'ouverture de son réseau social. La plupart des répondants ont déjà vécu des expériences de préjugés et stigmatisation qui les ont amenés à être vigilants dans le dévoilement ou encore à s'autocensurer. Ainsi, les jeunes adultes interviewés tendent majoritairement à dévoiler leur diagnostic de façon sélective. En d'autres mots, ils abordent le sujet auprès de personnes avec qui ils se sentent confortables, respectés et écoutés sans jugement. Les participants qui parlent ouvertement de leur diagnostic le font dans le but avoué de contrer les tabous et les préjugés véhiculés dans la société.

Parmi les impacts favorables liés au rapport aux autres dans le contexte de l'expérience de la maladie mentale, on constate que cette dernière peut parfois conduire au remodelage d'une image de soi plus positive. Les dires d'un participant qui s'est montré particulièrement optimiste quant à l'apport de la maladie mentale dans sa vie démontrent bien un tel cheminement identitaire : «Autant que j'ai pu creuser ma... bien ma propre tombe, c'est une manière de parler, autant que c'est moi qui a bâti l'échelle pour remonter du trou, si tu veux.» (P7). Ce participant mentionne qu'il a lui-même reconstruit son image de soi, minimisant ainsi l'impact du rapport aux autres. En lien avec son contexte personnel de vie, on constate qu'il a bénéficié et bénéficie toujours d'un soutien familial continu et de l'appui de son cercle d'amis. L'expérience singulière de la maladie peut également solidifier les rapports sociaux déjà existants ou favoriser les contacts, les rapprochements et les nouvelles opportunités sociales, tel que l'exprime ce participant : "Je sais pas parce qu'avec ma maladie, ça m’a aidé à rencontrer du monde, à faire toutes sortes d'affaires. Quand je pense, si j'avais pas eu de maladie, j'aurais pas eu ma famille ici 
"...pour un certain nombre de participants, l'expérience de la maladie a changé de manière constructive et satisfaisante leur rapport à l'autre et leur a permis de se renouveler en tant qu'êtres sociaux. "

\section{"La médication} est vue comme étant temporaire et passagère dans le parcours de ces jeunes adultes, alors que le diagnostic de maladie mentale, comme discuté précédemment, prend davantage une dimension permanente et intrinsèque à la personne. " [organisme communautaire], je sais pas. »(P6). Aussi surprenant que cela puisse paraître, pour un certain nombre de participants, l'expérience de la maladie a changé de manière constructive et satisfaisante leur rapport à l'autre et leur a permis de se renouveler en tant qu'êtres sociaux.

\section{Les services dans le champ de la santé mentale}

En ce qui a trait à la représentation et au positionnement des jeunes adultes par rapport au système de services de santé mentale, les propos sont variés. Dans l'approfondissement de ce sous-thème, plusieurs questions ont été abordées en lien, entre autres, avec la conception de la médication, la présence du discours médical, l'homogénéité des services selon le critère d'âge et les propositions de services de santé mentale. Parmi les positionnements identifiés, le traitement pharmacologique fait l'unanimité : il est aidant, mais non suffisant, à l'amélioration des symptômes de la maladie. Rodriguez et collab. (2001) vont dans le même sens et soutiennent que le traitement médicamenteux nécessite un contexte favorable d'utilisation. À cet effet, la majorité des participants croit fortement en la complémentarité de la pharmacothérapie et des services de nature psychosociale ou alternative. Nous avons par ailleurs observé une autre constante par rapport à la conception de la médication. La plupart des personnes interviewées souhaitent un jour arrêter définitivement ou, à tout le moins, diminuer la prise régulière de médicaments. La médication est vue comme étant temporaire et passagère dans le parcours de ces jeunes adultes, alors que le diagnostic de maladie mentale, comme discuté précédemment, prend davantage une dimension permanente et intrinsèque à la personne.

De toute évidence, les jeunes adultes interviewés reconnaissent l'utilité et l'importance des interventions et services psychiatriques. Cependant, quelques-uns remettent en question le bien-fondé de certaines pratiques, et ce, à la suite de malencontreuses expériences d'utilisation des services de santé mentale. L'expérience d'un participant au cours de laquelle son vécu affectif a été mis de côté pour laisser place à une attribution rapide et spontanée d'un diagnostic a suscité chez lui beaucoup d'incompréhension 
"Les interviewés suggèrent que les services tiennent compte à la fois de leurs défis en tant que jeunes adultes et de leurs difficultés associées à la maladie mentale. » et d'appréhension face au système psychiatrique. Cette situation illustre l'impact et la puissance du discours médical qui tend à évacuer le sens dans l'articulation explicative de la maladie mentale (Corin, 1993). Le discours médical possède justement des limites, auxquelles s'est heurté un autre participant. À la suite d'une hospitalisation d'urgence, celui-ci a été confronté à des pratiques médicales froides, expéditives et contraintes par le temps et l'espace.

Une certaine ambivalence s'exprime par rapport aux services de santé mentale. Les jeunes interviewés prônent les approches humaines et intéressées de la part des professionnels, mais dénoncent certaines mesures administratives qui réduisent l'accessibilité et la continuité des soins. Les opinions concernant la nécessité d'ajuster les services selon le critère d'âge sont mitigées, mais une même préoccupation demeure, soit l'importance de se sentir accueillis, compris et acceptés dans le partage de leur vécu. À partir de cette préoccupation majeure, plusieurs changements ont été proposés.

Les interviewés suggèrent que les services tiennent compte à la fois de leurs défis en tant que jeunes adultes et de leurs difficultés associées à la maladie mentale. Ils encouragent notamment les interventions de groupe spécifiquement pour les jeunes adultes, mais aussi une diversité d'interventions : suivis effectués par un mentor ou un coach de vie ${ }^{4}$, activités sociales diversifiées et facilement accessibles et ateliers de formation personnelle. Les démarches publiques de prévention et de sensibilisation avec une plus grande visibilité ont également été mentionnées, dans le but de pallier les effets pervers de la philosophie de "postvention »du système de santé actuel. Une participante a formulé à sa manière l'importance de diffuser de l'information liée aux transitions de vie et l'importance de la prévention de la maladie :

Il faudrait qu'on apprenne, au secondaire, comment faire face à la vie. Ça, on l'apprend pas. Qu'est-ce qui arrive quand on a des difficultés ou au niveau de la prévention, qu'est-ce qu'on fait pour éviter de tomber dans ces pièges-là? C'est quelque chose que j'aurais 
aimé savoir avant de tomber dans le piège de la maladie mentale, même étant quand même informée. (P3)

Tout comme cette participante, plusieurs se montrent en faveur de la promotion de la santé mentale sous toutes ses formes. Par le fait même, ils préconisent des campagnes publiques massives de sensibilisation à la maladie mentale et à la détresse psychologique.

\section{Discussion}

"La maladie mentale n'empêche pas les jeunes adultes de se définir comme des adultes, mais elle convie à une définition plus étoffée et plus large de ce qu'est être adulte, une définition faisant davantage référence à des repères personnels. »
À l'analyse des résultats précédents, nous dégageons certaines conclusions. Dans un premier temps, nous avons constaté que la maladie mentale ne représente pas qu'un frein dans la définition de soi comme adulte des jeunes adultes interviewés. Elle offre à ces jeunes d'autres repères d'ordre interpersonnel, professionnel, introspectif et prospectif. Ces nouveaux repères se conjuguent aux enjeux individuels et sociaux avec lesquels les jeunes adultes d'aujourd'hui doivent composer. La question de la perception de soi en tant qu'adulte nous a éclairé en ce sens. Le fait que les jeunes adultes interviewés se perçoivent en partie comme des adultes laisse à penser qu'ils se reconnaissent tout comme les jeunes adultes en général, une certaine autonomie, maturité ou sens des responsabilités et ce, malgré les impacts négatifs de la maladie sur l'image de soi ou la pleine jouissance des rôles sociaux. La maladie mentale n'empêche pas les jeunes adultes de se définir comme des adultes, mais elle convie à une définition plus étoffée et plus large de ce qu'est être adulte, une définition faisant davantage référence à des repères personnels. Parmi ceux-ci, notons le travail comme moyen d'expression et de réalisation de soi, l'importance de privilégier et de s'investir dans des rapports aux autres sains et réciproques, la valorisation du court terme, la maturité émotionnelle et la liberté comme des indicateurs du sentiment d'être adulte et les expériences vécues en tant qu'utilisateur de services dans le champ de la santé mentale. 
"...l'expérience de la maladie a précarisé le contexte personnel de vie des jeunes adultes, mais elle leur a aussi permis d'adopter une position critique et stratégique par rapport à leur statut d'adulte et à leur contexte de vie respectif."

"...la position qu'ont adoptée ces jeunes par rapport à leur réalité adulte et leur vécu psychiatrique, leur permet de se prononcer légitimement sur la qualité des services reçus et ainsi, de proposer de nouvelles orientations quant à l'offre de services actuelle."
Nous avons également constaté que l'expérience de la maladie mentale apporte chez ces jeunes adultes beaucoup de nuances dans la manière de concevoir leur réalité adulte. De toute évidence, l'expérience de la maladie a précarisé le contexte personnel de vie des jeunes adultes, mais elle leur a aussi permis d'adopter une position critique et stratégique par rapport à leur statut d'adulte et à leur contexte de vie respectif. Plusieurs d'entre eux posent un regard lucide et éclairé sur leur parcours de vie, sur les problèmes qu'ils rencontrent, sur leurs expériences professionnelles ou encore sur la manière de se projeter dans l'avenir (Desmarais et collab., 2000).

Ces constats nous permettent d'affirmer que l'image de soi des jeunes adultes ayant reçu un diagnostic de maladie mentale n'est pas nécessairement aussi négative que nous puissions le penser. L'image de soi de ces jeunes s'est préservée en partie grâce à l'émergence de nouveaux repères qui donnent un sens à ce qu'ils vivent. Que ce soit dans le rapport aux amis, dans le plaisir d'accomplir des tâches au travail ou dans la façon de vivre le moment présent, ces jeunes adultes entretiennent une image d'eux-mêmes plus positive, plus constructive. Bien que leur image de soi semble relativement préservée, il n'en demeure pas moins que la représentation qu'ils se font de la maladie et des services de santé mentale est déterminante dans l'analyse que nous avons réalisée. Nous nous devons par le fait même de souligner le positionnement réflexif de ces jeunes adultes et l'importance d'intégrer ce positionnement dans l'organisation des services dans le champ de la santé mentale (Kroes et Watling, 2009). En effet, la position qu'ont adoptée ces jeunes par rapport à leur réalité adulte et leur vécu psychiatrique, leur permet de se prononcer légitimement sur la qualité des services reçus et ainsi, de proposer de nouvelles orientations quant à l'offre de services actuelle. 


\section{Conclusion}

Le présent exercice d'analyse et de réflexion cherchait à aborder la maladie mentale dans une perspective non pathologisante et à élargir le cadre de compréhension que nous avons des phénomènes entourant les jeunes adultes d'aujourd'hui, en y intégrant l'expérience de la maladie mentale. L'éclairage porté ici sur la maladie mentale a mis en avant-plan l'expérience des jeunes adultes en tant que personnes et non, uniquement en tant que patient psychiatrique. Cette recherche visait ainsi globalement à déstigmatiser leur réalité, à déconstruire les a priori portant sur ces jeunes adultes et donc, à mieux comprendre les enjeux contradictoires auxquels ils sont confrontés (les exigences de performance, de productivité et d'autonomie versus des conditions de santé et de vie précarisées, fragilisées).

Outre l'intention d'améliorer la compréhension sociale et clinique de la problématique, nous souhaitons que soient revues les pratiques générales et les politiques sociales concernant les jeunes adultes vivant avec une maladie mentale. Nous aimerions ainsi stimuler chez le plus grand nombre d'acteurs, une réflexion critique portée sur l'état des pratiques et interventions destinées à ces jeunes adultes. De nombreuses questions peuvent se poser à cet égard.Est-ce que les propositions de services et les opinions émises par les jeunes adultes participant au projet pourraient s'insérer dans l'offre de services actuelle? Est-il possible de rendre la parole à ces jeunes adultes, afin de la placer au cœur des services qui leur sont destinés? En tant qu'intervenants, sommes-nous disposés à les entendre et à les traiter de sorte que leurs voix fassent écho au renouvellement, à la réorganisation et à la planification des services? Si oui, comment pourrions-nous y arriver?

\section{Notes}

Caroline Veilleux est récipiendaire de la Bourse Roland Lecomte 2009.

Cet article est basé sur les travaux menant à un mémoire de maitrise (Veilleux, 2009).

Les participants ont été recrutés sur une base volontaire par l'entremise de quatre organismes 
communautaires offrant des services en santé mentale dont deux sont situés à Gatineau, un à Ottawa et un à Montréal.Tous les participants devaient répondre aux critères d'inclusion suivants : être âgés de 18 à 35 ans, avoir reçu un diagnostic de maladie mentale (Axe I, DSM-IV-TR, 1994), avoir un suivi professionnel, vouloir partager son opinion et pouvoir s'exprimer en français.

$4 \quad$ Selon les propos des quelques participants ayant fait mention de cette suggestion, le coach de vie personnelle jouerait un rôle d'accompagnement et de soutien dans les démarches et le développement individuel de la personne.

\section{Bibliographie}

ALIE, Denis, Christine BOISVERT et Dany BRODEUR (2005). " Mobiliser les forces vives des jeunes aux prises avec des problèmes sévères ou transitoires de santé mentale ", réf. du 23 mars 2009, http://www.bibliotheque.assnat.qc.ca/01/MONO/2006/04/843995.pdf.

ANATRELLA, Tony (1999). "La postadolescence : quelques aspects psychologiques des 24-30 ans ", sous la direction de Poilpot, Marie-Paule, Un nouvel âge de la vie : le temps de la postadolescence, Ramonville Saint-Agne, Erès.

ANGENOT, Marc (1984). "Le discours social : problématique d'ensemble », Le discours social et ses usages, Cahiers de recherche sociologique, Vol.2, № 1, Avril, p. 19-44.

ANGENOT, Marc (1979). Glossaire pratique de la critique contemporaine, Ville LaSalle, Éditions Hurtubise HMH, Limitée.

ARNETT, Jeffrey Jensen (1998). «Learning to stand alone : the contemporary American transition to adulthood in cultural and historical context ", Human Development, Vol.41, № 5-6, p. 295-315.

ARNETT, Jeffrey Jensen (1997). «Young people's conceptions of the transition to adulthood». Youth and Society, Vol.29, No 1, p. 3-23.

BEAUJOT, Roderic, et Don KERR (2007). Nouvelles tendances dans les transitions chez les jeunes adultes au Canada, Gouvernement du Canada, Projet de recherche sur les politiques, Ottawa.

BIDART, Claire (2005). "Les temps de la vie et les cheminements vers l'âge adulte ", Lien social et Politiques, Vol.54, p. 51-63.

BIDDLE, Lucy, et collab. (2007). « Explaining non-help-seeking amongst young adults with mental distress : a dynamic interpretive model of illness behaviour ", Sociology of Health and Illness, Vol.29, $\mathrm{N}^{\circ}$ 7, p. 983-1002.

BIGGART, Andy, et Andreas WALTHER (2006). " Coping with Yo-Yo-Transitions. Young Adults' Struggle for Support, between Family and State in Comparative Perspective ", sous la direction de Leccardi, C. et Ruspini E., A New Youth? Young People, Generations and Family Life, Hampshire, Ashgate.

CAVALLI, Alessandro, et Olivier GALLAND (1993). L'allongement de la jeunesse, Poitiers, Actes Sud.

CHARBONNEAU,Johanne (2004). «Valeurs transmises, valeurs héritées », Les valeurs des jeunes, sous la direction de Pronovost, G. et Royer, C, Sainte-Foy, Les Presses de l’Université du Québec.

COLEMAN, James Samuel, et Torsten HUSÉN (1985). Devenir adulte dans une société en mutation, Paris, Organisation de Coopération et de Développement Économiques. 
CONTANDRIOPOULOS, André-Pierre, et collab. (1990). Savoir préparer une recherche : la définir, la structurer, la financer, Montréal, Presses de l'Université de Montréal.

CORIN, Ellen (1993). "Présentation. Les détours de la raison. Repères sémiologiques pour une anthropologie de la folie ", Anthropologie et Sociétés, Vol.17, No 1-2, p. 5-20.

DE SINGLY, François (2000). "Penser autrement la jeunesse », Lien social et Politiques, Vol.43, p.9-21.

DESMARAIS, Danielle, et collab. (2000). Détresse psychologique et insertion sociale des jeunes adultes. Un portrait complexe, une responsabilité collective, Ste-Foy, Les Publications du Québec.

DSM-IV-TR. (2004). Manuel diagnostique et statistique des troubles mentaux, Texte revisé, Guelfi, J.D., American Psychiatric Association, Paris, Elsevier Masson.

ESTROFF, Sue E. (1989). «Self, Identity, and Subjective Experiences of Schizophrenia : In Search of the Subject ». Schizophrenia Bulletin, Vol.15, № 2, p. 189-196.

GALLAND, Olivier (2004). Sociologie de la jeunesse, $3^{\mathrm{e}}$ édition, Paris, Armand Colin.

GALLAND, Olivier (1990). "Un nouvel âge de la vie ", Revue française de sociologie, Vol.31 № 4, p. 529-551.

GAUDET, Stéphanie (2005). «Responsabilité et identité dans les parcours d'entrée dans l'âge adulte : qu'est-ce que répondre de soi à l'âge adulte? ", The Canadian Review of Sociology and Anthropology, Vol.42, No 1 , p. 25-50.

GAUTHIER, Madeleine (2000). "L'âge des jeunes : un fait social instable », Lien social et Politiques, Vol.43, p. 23-32.

GAUTHIER, Madeleine (1994). Une société sans les jeunes?, Québec, Institut Québécois de recherche sur la Culture.

GENDRON, Benoît, et Jacques HAMEL (2004). "Travail, valeurs et être jeune : quel rapport? ", sous la direction de Pronovost, G. et Royer, C., Les valeurs des jeunes, Sainte-Foy, Les Presses de l'Université du Québec.

GLASER, Barney, et Anselm L. STRAUSS (1967). The Discovery of Grounded Theory, New York, Aldine.

GRALINSKI-BAKKER,J. Heidi, et collab. (2005). «Risks along the Road to Adulthood : Challenges Faced by Youth with Serious Mental Disorders ", sous la direction de Osgood, D. Wayne, E. Michael Foster, Constance Flanagan et Gretchen R. Ruth, On Your Own Without a Net, The Transition to Adulthood for Vulnerable Populations, Chicago, The University of Chicago Press.

HAMEL, J. (2000). «Je ne travaille pas, j'ai trop d'ouvrage. Pour de nombreux jeunes, le boulot ne sert qu'à payer le loyer et la croûte », sous la direction de Gauthier, Madeleine, Luce Duval, Jacques Hamel et Bjenk Ellefsen, Etre jeune en l'an 2000, Québec, Les Éditions de l'IQRC.

KROES, Geneviève, et Judy WATLING (2009). Transition saine vers l'âge adulte :Vers des soins intégrés en santé mentale, Rapport de l'atelier, Gouvernement du Canada, Projet de recherche sur les politiques.

L'ÉCUYER, René (1987). "L'analyse de contenu : notions et étapes », sous la direction de Deslauriers, Jean-Pierre, Les méthodes de la recherche qualitative, Sainte-Foy, Presses de l'Université du Québec, p. 54-63.

LECCARDI, Carmen (2006). "Facing Uncertainty. Temporality and Biographies in the New Century ", sous la direction de Leccardi, C. et Ruspini E., A New Youth? Young People, Generations and Family Life, Hampshire, Ashgate. 
MILES, Matthew B., et A. Michael HUBERMAN (2003). Analyse des données qualitatives, Traduction de la $2^{\mathrm{e}}$ édition américaine par Rispal, M. et révision scientifique de Bonniol, J. Paris, De Boeck.

MOLGAT, Marc (2007). «Do Transitions and Social Structures Matter? How Emerging Adults Define Themselves as Adults ", Journal of Youth Studies, Vol.10, № 5, November, p. 495-516.

MOLGAT, Marc (2000). "L'insertion résidentielle et les théories de la "modernité avancée". Quelques enseignements de la comparaison entre quatre sociétés ". Lien social et Politiques, Vol.43, p. 81-91.

MOLGAT, Marc (1999). " De l'intégration à l'insertion...Quelle direction pour la sociologie de la jeunesse au Québec? ", sous la direction de Gauthier, Madeleine, et Jean-François Guillaume, Définir la jeunesse? D'un bout à l'autre du monde, Saint-Nicolas, Les Presses de l'Université Laval.

NAKKULA, Michael J., et Eric TOSHALIS (2006). Understanding Youth. Adolescent Development for Educators, Cambridge, Harvard Education Press.

NELSON, Larry J., et Carolyn MCNAMARA BARRY (2005). «Distinguishing Features of Emerging Adulthood. The Role of Self-Classification as an Adult ". Journal of Adolescent Research, Vol.20, No 2, March, p.242-262.

NURMI, Jari-Erik (1999). "Self-Definition and Mental Health During Adolescence and Young Adulthood ", sous la direction de Schulenberg, John, Jennifer L. Maggs et Klaus Hurrelmann, Health risks and developmental transitions during adolescence, Cambridge, University Press.

PLUG, Wim, Elke ZEIJL et Manuela DU BOIS-REYMOND (2003). «Young People’s Perceptions on Youth and Adulthood. A Longitudinal Study from The Netherlands ", Journal of Youth Studies, Vol.6, No 2, p. 127-144.

POIRIER, Mario, et Jean GAGNÉ (1988) «Formes de l'appauvrissement et insertion sociale des jeunes adultes psychiatrisés ", Santé mentale au Québec, Vol.13, No 1, p. 132-143.

POULIN, Carole, et Raymond MASSÉ (1994). «De la désinstitutionnalisation au rejet social : point de vue de l'ex-patient psychiatrique ", Santé mentale au Québec, Vol.19, No 1, p. 175-194.

REITZLE, Matthias (2006). "The Connections between Adulthood Transitions and the SelfPerception of Being Adults in the Changing Contexts of East and West Germany ", European Psychologist, Vol.11, № 1, p. 25-38.

RODRIGUEZ, Lourdes, Mario POIREL et Ellen CORIN (2001). «Le point de vue des utilisateurs sur l'emploi de la médication en psychiatrie : une voix ignorée ", Revue québécoise de psychologie, Vol.22, No 2, p. 1-23.

ROULLEAU-BERGER, Laurence (1999). « Pour une approche constructiviste de la socialisation des jeunes ", sous la direction de Gauthier, Madeleine et Jean-François Guillaume, Définir la jeunesse? D'un bout à l'autre du monde, Sainte-Foy, Les Éditions de l'IQRC, Saint-Nicolas, Les Presses de l'Université Laval, p. 147-159.

SANTÉ CANADA (2002). Rapport sur les maladies mentales au Canada, Ottawa, Canada.

SCHEHR, Sébastien (2000). "Processus de singularisation et formes de socialisation de la jeunesse ", Lien social et Politiques, Vol.43, p. 49-58.

SHANAHAN, Michael J., et collab. (2005). Subjective age identity and the transition to adulthood. On the frontier of adulthood. Theory, Research and Public Policy, sous la direction de Setterstein, Richard A. Jr., Frank F. Furstenberg Jr. et Rubén G. Rumbaut, Chicago, The University of Chicago Press. 
SHULMAN, Shmuel, et collab. (2009). "Self-regulatory processes and psychological symptoms among emerging adults ", Journal of Youth Studies, Vol.12, № 1, February, p. 111-120.

VEILLEUX, Caroline (2009). "Que signifie être jeune adulte aujourd'hui? » : Point de vue de jeunes adultes ayant reçu un diagnostic de maladie mentale, Mémoire déposé à l'École de service social, sous la direction de Marc Molgat, Université d'Ottawa.

WESTBER G, Annika (2004). "Forever Young? Young People's Conception of Adulthood : The Swedish Case ", Journal of Youth Studies, Vol.7, № 1, March, p. 35-53. 